\title{
ONOMÁVAEIN
}

Journal of linguistics, philology and translation

\section{La correction phonétique de la prononciation du français dans un contexte universitaire Jordanien}

Phonetic correction of pronunciation of French language in Jordanian

university context

\section{Rana Hasan Kandeel \\ Yarmouk University \\ Jordan}

\section{Virginie Marcy}

Alliance Française de Minneapolis-Saint Paul

USA

\section{(C) $\underset{\mathrm{BY}}{\ominus}$}

Rana Hasan Kandeel: Department of Modern Languages, Yarmouk University, Irbid, Jordan. | E-mail: ranakand@yahoo.fr

Virginie Marcy: Alliance Française de Minneapolis-Saint Paul, Minnesota, USA. | E-mail:vinivicci@gmail.com 


\section{Résumé}

Cet article a pour objectif de présenter les approches adoptées dans l'enseignement de la prononciation en français langue étrangère (FLE). L'identification des difficultés de prononciation, le travail sur sa correction dans les phases de remédiation et de post-remédiation constituent les étapes essentielles de la méthodologie de l'enseignement. Pour mettre en œuvre cette méthodologie, nous avons procédé à analyse contrastive des systèmes vocaliques et consonantiques de la langue cible (le français) et de la langue maternelle (l'arabe standard), ainsi qu'à la mise en place d'exercices et d'activités ciblées et des tests d'évaluation à la fin du parcours d'apprentissage. L'étude que nous présentons se concentre sur la consonne oc-

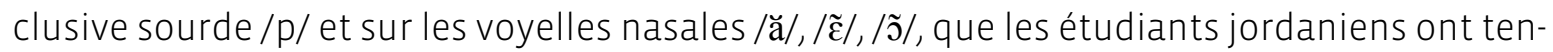
dance à confondre. Les résultats de cette expérimentation montrent que l'amélioration de la prononciation est possible. II faut donc que les enseignants fassent de la correction phonétique en classe avec les apprenants, surtout débutants, après avoir relevé soigneusement leurs besoins dans ce domaine.

Mots clés: prononciation ; correction phonétique ; français langue étrangère (FLE) ; démarches pédagogiques; langue arabe.

\section{Abstract}

This article aims to present the approaches adopted in teaching pronunciation in French as a foreign language (FFL). The identification of difficulties of the pronunciation, the work on its correction in the phases of remediation and post-remediation are the essential steps of teaching methodology. To implement this methodology, we performed a contrastive analysis of the vocal and consonant systems of the target language (French) and the mother tongue (standard Arabic), as well as the implementation of exercises, activities, and assessment tests at the end of the learning. The present study focuses on the deaf /p/ occlusive consonant and the nasal vowels $/ a ̆ /, / \varepsilon /, / \tilde{\partial} /$, which Jordanian students tend to confuse. The results of this experiment show that improvement of pronunciation is possible. Teachers must therefore make phonetic correction in class, with learners, mostly beginners, after carefully recording their needs in this area.

Keywords: pronunciation; phonetic correction; French as a foreign language (FFL); pedagogical approaches; Arabic language. 


\section{Introduction}

L'importance de la phonétique dans un cours de français langue étrangère n'a plus besoin de justification. Dans la didactique de l'oral, la phonétique joue un rôle essentiel. Pour se faire comprendre et comprendre son interlocuteur, il faut une prononciation et une audition de qualité pour qu'il n'y ait pas d'ambiguïté dans la réception du message. Pour les apprenants d’une langue étrangère, la prononciation des mots ou des phrases se fait aisément pour les phonèmes transférables de leur langue maternelle dans la langue étrangère. Mais les apprenants commettent parfois de nombreuses erreurs du fait que certains phonèmes de la langue étrangère n'existent pas dans leur langue maternelle. L'enseignant doit les repérer, les leur signaler et introduire des méthodes de correction phonétique.

La question qui nous intéresse ici est celle-ci : comment corriger efficacement la prononciation du français des étudiants jordaniens apprenant le FLE dans un cours de phonétique en licence de langue française à l'université du Yarmouk en Jordanie ? L'efficacité signifie la proposition des séances de travail sur des exercices et des activités complètement conçues en fonction des erreurs de prononciation commises par les apprenants et en vue de l'amélioration de la prononciation. Notre objectif est de faire progresser l'acquisition de la prononciation du français en adoptant une démarche pédagogique fondée sur l'identification des difficultés, le travail de remédiation et la phase de post-remédiation sachant que les difficultés de prononciation ne sont pas les mêmes pour tous les apprenants de FLE, les systèmes phonologiques étant différents d'une langue à l'autre. La démarche pédagogique proposée dans le cadre de cette recherche doit fournir des consignes simples et un langage accessible aux enseignants dans la correction des erreurs afin de faire le pont entre la recherche théorique et son application dans la pratique quotidienne. II s'agit d'une implication pratique des notions théoriques en prononciation.

\section{La phonétique et la didactique des langues}

La production orale comporte des aspects linguistiques (phonétique, lexical, grammatical, orthographique), des aspects pragmatiques (la fluidité, les registres de langue, le rythme de la parole) et des aspects expressifs (l'intonation expressive, les mimiques, la posture et la gestuelle). Les méthodes récentes de FLE tentent de varier les activités phonétiques afin d'encourager l'étude de la prononciation, le plus souvent jugée ennuyeuse par les apprenants (Cuq \& Gruca, 2005). Pour les didacticiens de langue, la prononciation constitue la "manière de produire, de réaliser les phonèmes d'une langue et les traits prosodiques qui, dans la chaîne parlée, accompagnent la réalisation des phonèmes" (Galisson \& Coste, 1976).

La phonétique reste le premier obstacle à surmonter dans l’apprentissage de cette langue. Vaincre cet obstacle est essentiel car elle est "à la base de l'acquisition d'une compétence de 
communication et conditionne en tout premier lieu la compréhension et l'expression orales" (ibid.). L'enseignement de la phonétique en FLE est indispensable car la langue est avant tout "une matière sonore, (...) elle est une composante essentielle du langage avec le lexique, la grammaire et le code orthographique et (...) la sensibilisation aux phénomènes phonétique (...) favorise la compréhension et la production de la langue étrangère" (Abry \& Chalaron, 2010).

La qualité d'une communication et la compréhension des interlocuteurs dans un échange dépendent largement de la prononciation parce que "la compétence de communication, que ce soit en compréhension de l'oral ou en production orale, repose sur une série de distinctions phonologiques sur lesquelles vont s'élaborer des distinctions grammaticales et lexicales" (Abry \& Veldeman Abry, 2007 : 7). En effet, le décalage entre les formes de l'oral et les contraintes orthographiques (Paveau \& Sarfati, 2003) incite tout chercheur et enseignant à mener une réflexion importante non seulement sur la correction des erreurs de prononciation, mais également sur la formation des apprenants à la représentation graphique de la parole par l'Alphabet Phonétique International (API).

Le cadre européen commun de référence pour les langues (CECRL, 2000 : 73-74) a redéfini la compétence de communication. La composante linguistique a trait aux savoirs et savoir-faire relatifs au lexique, à la syntaxe, à la phonologie et à l'orthographe. Cette définition revalorise l'apprentissage de la phonétique dans une classe de langue et place les aptitudes phonétiques et la compétence phonologique au centre des préoccupations des enseignants de langues.

L’apprenant doit être capable de parler, écrire, écouter et lire. Pour communiquer dans une langue étrangère, il a besoin des aptitudes phonétiques essentielles à la prononciation et à l'audition d'un énoncé (CECRL, 2000 : 85) telles que :

- La capacité d’apprendre à distinguer et à produire des sons inconnus et des schémas prosodiques.

- La capacité de produire et enchaîner des séquences de sons inconnus.

- La capacité, comme auditeur, de retrouver dans la chaîne parlée la structure significative des éléments phonologiques (c'est-à-dire de la diviser en éléments distincts et significatifs).

- La compréhension et maîtrise du processus de réception et de production des sons applicable à tout nouvel apprentissage d'une langue.

Pour acquérir une compétence phonologique, l'apprenant doit percevoir et produire certains éléments phonologiques, comme les unités sonores de la langue (les phonèmes), les traits distinctifs des phonèmes (la labialité, la sonorité, la nasalité, etc.), la composition phonétique des mots (par exemple la structure syllabique), la suite des phonèmes, l'accentuation des mots, l'assimilation, les allongements, le rythme, l'intonation linguistique et expressive. La maîtrise 
de la compétence phonologique est délimitée par le CECRL selon les différents niveaux de langues (tableau 1).

\section{TABLEAU 1}

Compétence phonologique selon le CECR

\begin{tabular}{cl}
\hline A1 & $\begin{array}{l}\text { La prononciation d’un répertoire très limité d'expressions et de mots } \\
\text { mémorisés est compréhensible avec quelque effort pour un locuteur natif } \\
\text { habitué aux locuteurs du groupe linguistique de l'apprenant/utilisateur. }\end{array}$ \\
\hline A2 & $\begin{array}{l}\text { La prononciation est en général suffisamment claire pour être comprise malgré } \\
\text { un net accent étranger mais l'interlocuteur devra parfois faire répéter. }\end{array}$ \\
\hline B1 & $\begin{array}{l}\text { La prononciation est clairement intelligible même si un } \\
\text { accent étranger est quelquefois perceptible et si des erreurs } \\
\text { de prononciation surviennent occasionnellement. }\end{array}$ \\
\hline B2 $\quad \begin{array}{l}\text { L'apprenant a acquis une prononciation et une intonation claires et naturelles. } \\
\text { C1 }\end{array} \quad \begin{array}{l}\text { L'apprenant peut varier l'intonation et placer l'accent phrastique } \\
\text { correctement afin d'exprimer de fines nuances de sens. }\end{array}$ \\
\hline C2 & Comme C1
\end{tabular}

L'enseignant doit décider les aptitudes phonétiques à exiger des apprenants en tenant compte de leurs niveaux linguistiques et des objectifs d'apprentissage. II doit encourager l'apprenant à adopter une nouvelle prononciation tout en l'associant au plaisir de l'écoute de mots, de documents sonores amusants et de mélodies attrayantes. Mais l'enseignement de la langue française par un enseignant natif aux apprenants arabes impose une connaissance du fonctionnement de la langue arabe (Maume, 1973) pour éviter une attitude de condamnation des fautes commises dans la prononciation sans en savoir les origines. Il est aussi important de préciser que l'objet de notre étude est la correction de l'erreur de prononciation et non pas les fautes de prononciation. Selon Corder (1980), l'erreur renvoie à des lacunes dues à la connaissance sous-jacente de la langue étrangère par l'apprenant contrairement à la faute qui désigne les lacunes dues en langue étrangère aussi bien qu'en langue maternelle à des défaillances de mémoire, à des états physiologiques tels que la fatigue ou les émotions fortes. L'erreur selon la première définition est systématique et elle permet de reconstruire la compétence transitoire de l'apprenant. Dans ce travail de recherche, nous nous intéressons aux erreurs systématiques des apprenants jordaniens étant donné que ces erreurs se répètent et elles manifestent ce qu'ils ont appris dans le système linguistique du français.

La connaissance du système phonologique français par un enseignant non natif est une exigence pour comprendre les différences entre les systèmes phonologiques des deux langues en présence, le français et l'arabe, ce qui permettra aux enseignants de connaître "le fondement de transferts positifs ou négatifs de traits propres à des langues déjà acquises par l'apprenant vers la langue-cible" (Blanchet, 2002 : 47). 
Cette approche contrastive permetà l'enseignant d'anticiper les interférences et de diagnostiquer les difficultés de prononciation afin de travailler les points faibles. Il peut ainsi rectifier la prononciation erronée qui peut gêner la compréhension d'un message. La correction phonétique doit exploiter les résultats de l'analyse contrastive entre la langue maternelle et la langue cible.

\section{Analyse contrastive des systèmes vocaliques et consonantiques du fran- çais et de l'arabe standard}

Le français compte dix-huit consonnes phonétiques et seize voyelles dans ses systèmes consonantique et vocalique alors que l'arabe standard comprenne vingt-huit consonnes et six voyelles dont trois longues et trois courtes. Saidane et al. (2004) proposent une classification des consonnes en arabe en fonction de critères essentiels tels que la vibration ou pas des cordes vocales lors de l'articulation et le franchissement de l'air à travers le conduit vocal produisant des variétés de sons. Quant aux voyelles, courtes ou longues, elles sont caractérisées par la vibration des cordes vocales mais leur durée ne sont pas les mêmes. Dans la comparaison de ces deux systèmes, les paramètres à retenir sont ceux qui s'ordonnent autour des traits comme occlusive/constrictive; nasale/orale; sourde/sonore; vélaire/palatale.

Une synthèse de la comparaison entre les systèmes vocaliques et consonantiques français et arabe permet de constater les points suivants:

\subsection{Les systèmes vocaliques français et arabe}

a) L’aperture ou la hauteur de la langue est plus développée en français : quatre niveaux pour deux en arabe.

b) La nasalité n'existe pas en arabe.

c) La durée, liée à l'accentuation, est un critère pertinent en arabe. En français, la durée n'est pas pertinente. Néanmoins, selon son entourage consonantique et sa position en syllabe accentuée ou non accentuée, la voyelle peut parfois varier en durée.

Toutes les voyelles nasales sont longues en syllabes fermées (content > contente).

Toutes les voyelles orales sont longues devant les consonnes [r], [v], [z], [3], [vr] (père, rêve, aise, beige, lèvre).

d) La glottalisation en arabe : le coup de glotte (hamza) se trouve à l'initiale, à l'intérieur d'un mot ou en finale. Elle se note [?] ; le hamza ne modifie pas le son de la voyelle précédente; il est compté comme une syllabe; il n'existe pas en français.

e) Les voyelles françaises sont nombreuses (13) et leur réalisation phonétique peu variable alors que les voyelles arabes sont au nombre de trois produisant les sons vocaliques sui- 
vants : "i" la plus fermée à l'avant, "a" la plus ouverte, "ou" la plus fermée à l'arrière. Ces voyelles connaissent des réalisations phonétiques très étendues selon leur environnement consonantique de telle sorte que les signes diacritiques qu'on leur associe produisent des sons intermédiaires [mat], [maot], [mita].

f) En arabe, l'arrondissement des lèvres et leur projection s'appliquent uniquement à la voyelle [u] alors qu'en français huit sont concernées, avec notamment une série de trois voyelles arrondies antérieures.

\subsection{Les systèmes consonantiques français et arabe}

a) Les consonnes arabes sont plus nombreuses qu'en français et ont des lieux d'articulation spécifiques qui n’existent pas en français:

- interdentales $[\theta],[ð],\left[z^{\varsigma}\right]$;

- pharyngales [ћ], [? $\left.{ }^{\mathrm{c}}\right]$;

- glottale [ћ].

b) Les consonnes arabes ont un mode d'articulation spécifique : les emphatiques $\left[\mathrm{d}^{\varsigma}\right],\left[\mathrm{s}^{\varsigma}\right]$, $\left[\mathrm{t}^{\mathrm{c}}\right],\left[\mathrm{z}^{\mathrm{q}}\right]$.

c) En arabe toutes les consonnes peuvent être doublées ou géminées : la gémination est indiquée par un signe diacritique appelé "chadda" qui change le son des mots ([aba/ et [abba/) ; en français la gémination est seulement de position, exemple : il a dit / il l'a dit.

d) Les consonnes françaises [p], [v], [n] n'existent pas en arabe.

e) Les trois semi-consonnes [w], [u], [j] n'existent pas en arabe.

\section{L'état de l'art}

L'analyse des difficultés de la prononciation des apprenants arabophones étudiant le français est abordée dans de nombreuses recherches. En respectant les variations linguistiques influant sur la prononciation, les chercheurs arabophones ont analysé les difficultés selon leur public cible. Ainsi, les travaux d'Al-Tamimi, Girard et Marsico (2002) et d'Al-Tamimi (2006, 2007) sur des apprenants jordaniens et ceux d'Al-Shihri (2002) sur des étudiants saoudiens et ceux de Yousif (1999) sur des apprenants qataris ont abordé les difficultés de la production et de la perception des voyelles orales du FLE chez un arabophone. Mais l'objectif de ces recherches était de mener plutôt une analyse contrastive afin d'identifier les sources des difficultés sans adopter des approches de correction phonétique pour ce public.

Pour la correction phonétique, nous citons les travaux de Cheddad (2011) sur des apprenants algériens dans le cycle primaire et ceux d'Abdessemed et Abdelhanmid (2015), qui ont 
développé un didacticiel de correction phonétique pour des apprenants algériens basé sur l'analyse d'enregistrement de variantes phonétiques. La première étude a visé des élèves en premier cycle, alors que notre travail porte sur des étudiants à l'université. Quant à la deuxième étude, en se concentrant sur l'analyse des erreurs des apprenants dans la prononciation, le système ne propose que la correction au niveau des mots isolés français sans travailler la correction des sons au niveau de la phrase ou proposer des activités contextualisant la prononciation comme la lecture.

Cette limite de la correction phonétique par la machine, et d'autres limites telles que l'absence d'analyse de la gestualité associée à la production sonore ont déterminé notre choix de présenter une démarche humaine et pédagogique de correction phonétique. Toutes ces recherches ont réussi à diagnostiquer les difficultés de la prononciation mais sans proposer une démarche cohérente de correction phonétique pour un public jordanien. Dans notre recherche, il s'agit de faire face à la question de délaissement de l'enseignement de la prononciation car celle-ci est perçue comme une matière difficile à enseigner et à évaluer par l'enseignant (Wachs, 2011). Pour l'apprenant jordanien du FLE, il a besoin d'une explication explicite des règles de prononciation et des relations entre les phonèmes et les graphèmes dans un cadre pédagogique d'activités et d'exercices bien réfléchis afin de bien prononcer et communiquer en français. L'étude présente s'appuie essentiellement sur les travaux des spécialistes dans ce domaine (cf. Abry, 2008 ; Abry \& Chalaron, 2010).

\section{L’apprentissage de la phonétique française dans le contexte universitaire jordanien}

Le travail sur la prononciation et l'audition des sons français n'est pas assez mis en valeur dans le contexte universitaire. Ce travail se fait dans les cours en suivant la progression proposée dans les manuels FLE. Les apprenants de niveau débutant Al (première année) travaillent la prononciation des sons dans le laboratoire de langues à travers, essentiellement, des exercices de répétition de phrases isolées.

\subsection{Pratique de la prononciation en français}

La pratique de la prononciation en classe est très rare du fait que l'oral est peu pratiqué dans les méthodes d'enseignement adoptées dans notre contexte et que les apprenants ont très peu l'occasion de communiquer avec des natifs à l'extérieur. Le manque de pratique linguistique en classe (et à l'extérieur de la classe évidemment) ainsi que le manque de correction phonétique font que certains étudiants se retrouvent parfois en deuxième ou troisième année d'études avec des lacunes de prononciation qui auraient dû être comblées bien plus tôt dans leur parcours universitaire. De plus, en Jordanie, certains professeurs ont tendance à 
enseigner leur matière comme on le fait avec des apprenants français qui possèdent déjà les structures et la prononciation du français de base.

Un cours de phonétique est enseigné à la fin de la deuxième année d'étude à l'Université du Yarmouk. Cependant la matière est facultative et les élèves peuvent choisir de ne pas l'étudier. Ce cours semestriel de 15 semaines (la classe a lieu trois fois par semaine pour une durée de 50 minutes) a pour but d'initier les apprenants à quelques notions phonétiques de base, car "de longues années de travail sont (...) nécessaires pour parfaire la prononciation : rendre les sons fluides et acquérir la prosodie française, jusqu'à ce que tout devienne une sorte d'automatisme" (Sung, 2002 : 203). Le cours de phonétique de l'Université du Yarmouk cherche également à expliquer la relation entre le son et les lettres de la langue française afin d'acquérir une prononciation, un rythme et une lecture à voix haute proche de celle des Français. Le cours présente une classification des sons du français et leur prononciation exacte.

\subsection{Langue d'instruction dans le cours de phonétique}

La classe est enseignée par une native qui parle uniquement français, ce qui donne une démarche plus authentique à l'apprentissage des sons. Les étudiants "en étant plongé dans le bain rythmique du français, (...) s'imprègne[nt] de l'originalité rythmique du français parlé en développant des automatismes comme c'est le cas en langues maternelle" (Pagel et al., 2012). Le choix d'une Française pour ce cours n'a rien d'hasardeux. Derrière cette décision, il y a la volonté que les étudiants parviennent à entendre, observer et imiter l'accent du professeur.

\section{Echantillon des sujets et objet de l'étude}

Notre échantillon est formé d'un groupe de 20 étudiants inscrits dans le département de langue moderne de l'Université du Yarmouk. Leur niveau de maîtrise phonologique se situe entre A2 et B1. Ils préparent une licence de français et ont déjà suivi environ 115 heures de français. Ce sont de jeunes adultes âgés de 20 à 25 ans. Cette classe a été enseignée à quatre reprises au cours de quatre semestres différents. Seul le dernier semestre d'enseignement, et le groupe d'étudiant correspondant a été pris en compte pour cette étude. Au cours des trois semestres d'enseignement de cette classe de phonétique, il a été observé que certains exercices phonétiques étaient plus probants que d'autres pour le compte de notre étude. Ces exercices ont été choisi et seront présentés dans cette étude.

Notre objet d'étude est constitué de l'ensemble de réalisations phonétiques du français oral produites par ces étudiants au moment de la communication avec le professeur et de la réalisation des activités pédagogiques en classe telles que la lecture et la réalisation des exercices et des activités communicatives à l'oral. En effet, notre audition attentive des sons 
focalisée sur les points précis de disfonctionnement de la prononciation des sons et l'analyse contrastive de deux systèmes phonologiques français et arabe ont montré que la nasalité ainsi que le son /p/n'existant pas en arabe constituent entre autres les principales difficultés de prononciation pour notre public. Pour cette étude, nous nous focaliserons sur la consonne occlusive sourde /p/ que les étudiants jordaniens prononcent quasiment comme la sonore

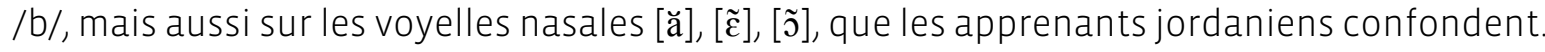

\section{Le travail de correction phonétique mis en place}

Tout au long de l'année les étudiants ont été écoutés en grand groupe puis en petit groupe ainsi qu'en entretien individuel à la fin du semestre pour évaluer les résultats du travail effectué en phonétique.

Afin de corriger la prononciation de nos étudiants, plusieurs démarches de correction phonétique ont été adoptées. Nous avons utilisé la méthode articulatoire. Nous avons commencé par des exercices de reconnaissance auditive, nous avons effectué ensuite des exercices de prononciation de sons en opposition dans des mots et des phrases, en position accentuée et inaccentuée pour les voyelles et dans toutes les positions pour les consonnes.

Nous avons également travaillé la lecture de textes. Les exercices choisis seront détaillés pour chaque phonème posant problème. Le travail de correction a continué tout le semestre. II s'est déroulé selon l'emploi du temps suivant : évaluation initiale et observation du professeur: une semaine ou trois séances, reconnaissance auditive trois semaines ou neuf séances, exercice de prononciation dix semaines ou trente séances, évaluation finale une semaine ou trois séances. Le temps de travail sur chaque individu était variable du fait du niveau hétérogène de la classe.

\subsection{Les consonnes /p/ et /b/}

La première difficulté constatée concerne l'opposition /p/, /b/. Ces deux sons présentent des caractéristiques communes. Ce sont deux occlusives bilabiales, les lèvres se ferment et appuient nettement l'une contre l'autre et il se produit une explosion brutale brève. A cela s'ajoute le fait que ces consonnes ne peuvent pas être prolongées. On les appelle des momentanées. Dans les deux cas, le voile du palais est relevé.

La différence porte sur la vibration des cordes vocales, c'est-à-dire sur l'opposition sourde/sonore. En effet, /p/ est une consonne sourde et les cordes vocales ne vibrent pas. Au contraire, /b/ est une consonne sonore et les cordes vocales vibrent. Mais comme le son /p/ n'existe pas en arabe, il tend à être confondu avec le /b/. Pour aider les étudiants à savoir 
la différence entre ces deux consonnes, Abry (2008) propose de partir "du constat que les sourdes sont toujours plus tendues que les sonores correspondantes. Si on dit 'pa' et 'ba' dans la paume de sa main le 'pa' fera beaucoup plus d'air". Pour accentuer cette tension, on privilégiera de plus les mots commençant par/p/, comme "Paris" ou "poulet".

Nous avons proposé un exercice de discrimination auditive où la consonne est au début du mot. Les étudiants devaient cocher ce qu'ils entendaient. Pour le premier exemple (tableau 2), les étudiants devaient cocher la case bas (Abry \& Chalaron, 2010).

\section{TABLEAU 2}

Exercice présentant les phonèmes/p/ et /b/ au début du mot

\begin{tabular}{|c|c|c|c|}
\hline Pas & & Bas & $\checkmark$ \\
\hline Pou & $\ldots$ & Bout & $\ldots$ \\
\hline Pont & $\ldots$ & Bon & $\ldots$ \\
\hline Peau & $\ldots$ & Beau & \\
\hline Pelle & $\ldots$ & Belle & \\
\hline
\end{tabular}

Les étudiants n’ont eu aucune difficulté à réaliser cet exercice. Ils perçoivent la différence entre /p/ et /b/. Ensuite, il a fallu s'assurer également que les apprenants ne confondaient pas les deux consonnes en milieu de mot (happé/abbé) et à la fin (trompe/trombe). Ces mots ont été répété en grand groupe puis en plus petits groupes, enfin de manière individuelle. II faut noter que la répétition collective est une méthode qui rassure les étudiants et permet aux timides de s'engager plus facilement. Lorsque les mots sont articulés séparément, les étudiants n'éprouvent aucune difficulté à distinguer les deux consonnes. Nous avons aussi travaillé la prononciation en français de paires minimales dans toutes les positions. Mais une bonne perception ne suffit pas pour produire une articulation exacte de ces sons. II est nécessaire de faire pratiquer ensuite une série d'exercices de prononciation.

Une fois la bonne audition est acquise, nous sommes passés à la prononciation des mêmes exercices où les sons /p/ et /b/ surtout en position finale, qui est la plus difficile à acquérir car c'est une position faible. Les étudiants devaient bien faire "exploser" le /p/ ou le /b/ :

$$
\begin{aligned}
\text { Exemple: } & \text { Ça tape } & \text { Quelle jolie robe } \\
& \text { Allez hop! } & \text { J'ai mal àla jambe }
\end{aligned}
$$

Pour notre groupe d'étudiant, la consonne sonore, qui est plus faible que la sourde, était plus facile à réaliser dans ce cas. Cependant, nous avons insisté sur l'explosion du son à la finale - l'exercice est excellemment réalisé lorsqu'un objectif est donné - , puis nous avons continué avec une articulation plus "normale", plus naturelle et plus difficile. 
Lorsque les étudiants semblaient compétents pour différencier ces deux sons, nous les avons mis en contexte dans des phrases un peu plus longues, qui comportaient plusieurs fois les sons étudiés:

Le petit bébé a sali son bavoir avec du biscuit.

La pauvre Brigitte a perdu son passeport au bazar.

Est-ce qu'il y a du porto à Bordeaux?

Nous avons fait écouter ces enregistrements aux étudiants et ils devaient les répéter ensuite. Les enregistrements de différentes voix de natifs extraits des CD audio provenant de livres cités en bibliographie ont également été utilisés souvent afin de renforcer l'oreille des apprenants avec un bain linguistique prenant en compte la variation tonale. Il est préférable que les étudiants répètent ces phrases sans avoir l'écrit sous les yeux afin que la graphie n’influence pas la prononciation du son.

Après tous ces exercices, le bilan a été concluant, les apprenants ont eu des difficultés mais sont arrivés à prononcer le /p/. Pour la majorité des étudiants (treize en tout), nous avons remarqué que la prononciation du /b/ a été plus accentuée dans des mots tels que "observer". Nous avons expliqué que ce mot est un cas particulier en français car le /b/ se prononce comme /p/à cause du /s/, mais qu'en anglais, la première langue étrangère apprise par nos étudiants, le /b/ est prononcé /b/ mais le /s/ se sonorise en /z/ à cause du/b/.

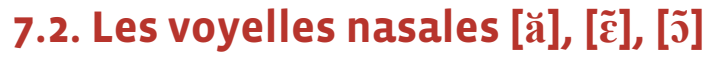

Ces trois voyelles nasales n'existent pas en arabe. Pour ces trois sons, le voile du palais est abaissé et les cordes vocales vibrent. Les différences résident dans le fait que:

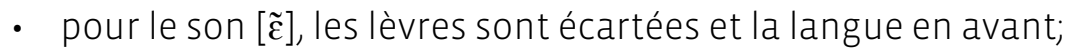

- pour le son [ă], les lèvres sont un peu arrondies, la langue est en arrière et plus basse que pour les deux autres voyelles nasales;

- pour le son [̃̃], les lèvres sont arrondies, projetées et fermées, la langue est en arrière et en position assez basse comme pour la voyelle $[\tilde{\varepsilon}]$.

Les étudiants jordaniens ont du mal à prononcer ces trois voyelles qu'ils perçoivent comme une voyelle orale.

Notre travail a commencé par une sensibilisation à la nasalité. Nous avons montré aux étudiants des coupes sagittales représentant le chemin pris par l'air ainsi que la position des organes (Abry \& Chalaron, 2010). Pour certains étudiants ce fut une théorie concluante, pour 
d'autres il a fallu utiliser un aspect plus "pratique" afin qu'ils ressentent corporellement ce qui se passait dans la bouche: nous avons effectué une pression sur le nez avec les doigts afin de

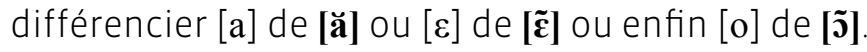

Le travail de reconnaissance auditive a ensuite commencé. Comme il est plus aisé pour les étudiants d'entendre une voyelle longue qu'une voyelle brève, un son accentué qu'un son inaccentué, nous avons commencé par un premier exercice de distinction où la voyelle est en syllabe fermée accentuée (tableau 3).

\section{TABLEAU 3}

Exercice présentant les voyelles nasales en syllabe fermée accentuée

\begin{tabular}{|c|c|c|}
\hline 1. $[\tilde{\varepsilon}]$ & 2. [ă] & 3. [5] \\
\hline Pince & Pense & Ponce \\
\hline Teinte & Tente & Tonte \\
\hline Mainte & Menthe & Monte \\
\hline Feinte & Fente & Fonte \\
\hline
\end{tabular}

Les apprenants devaient d'abord cocher le mot entendu, puis ils ont entendu les trois mots dans un ordre aléatoire et ils devaient déterminer l'ordre réalisé : 123, 213 ou 321 ? Pour sept étudiants, la distinction entre le son [ă] et [0̃] demeure un problème, il fut nécessaire d'aller plus loin dans l'apprentissage.

Nous avons donc ensuite travaillé la discrimination des voyelles nasales en syllabe ouverte (tableau 4).

\section{TABLEAU 4}

Exercice présentant des voyelles nasales en syllabe ouverte

\begin{tabular}{|c|c|c|}
\hline$[\tilde{\varepsilon}]$ & [ă] & 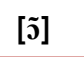 \\
\hline Lin & Lent & Long \\
\hline Sain & Sang & Son \\
\hline Teint & Temps & Ton \\
\hline Daim & Dent & Dont \\
\hline
\end{tabular}

Nous avons pratiqué aussi une autre variante de ces exercices de discrimination. Trois mots comportant les trois voyelles nasales étaient prononcés et un quatrième mot était ajouté, il reprenait un de ces mots (tableau 5), les étudiants devaient souligner celui qui avait été répété (Abry \& Chalaron, 2010). 


\section{TABLEAU 5}

Exercice de discrimination des voyelles nasales

\begin{tabular}{llllll}
\hline Exemple & Frein & Franc & Front & $\rightarrow$ & {$[$ frẽ $]$} \\
\cline { 2 - 6 } & Main & Ment & Mon & $\rightarrow$ & {$[$ mă] } \\
\hline
\end{tabular}

Il n’y a pas eu de difficulté majeure quant à la réalisation de ces derniers exercices. Un autre exerciceplus classique permet de contrôlersiles étudiants ont bien intégréla différenceentreles trois voyelles nasales. Il leur est demandé de transcrire phonétiquement sous la dictée des phrases comportant les trois phonèmes. Il a fallu plus d'un essai pour qu'ils parviennent à réussir cette transcription. Seul quatre étudiants sont parvenus à retranscrire parfaitement cette phrase.

Exemple: Donnez-moi un bon vin blanc.

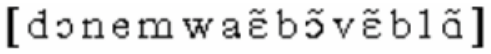

Après l'audition acquise, nous nous sommes concentrés sur un travail d'entraînement articulatoire avec les mêmes exercices, mais cette fois en prononciation. Nous avons remarqué que la timidité de certains étudiants empêchait le professeur de bien entendre le son prononcé, mais nous leur avons demandé de parler plus fort. Pour améliorer la prononciation, le professeur peut utiliser l'allongement de la voyelle et exiger que la durée du son soit exagérée. Ainsi, il entend mieux si le son est prononcé correctement et le côté ludique peut amuser les apprenants. II ajoute le relâchement articulatoire en demandant aux apprenants de se détendre en baissant les épaules.

Exemple: "Je peeeeeeeeeennnnnnnnnnnnse qu'il n’est pas là"; "Mooooooooooonnnnnnnte dans la voiture".

Le professeur peut aussi chercher ces sons dans des chansons françaises, et c'est l'entraînement sur le travail en voix chantée qui apporte des avantages à ne pas nier au niveau des capacités articulatoires et perceptives des apprenants. Cornaz et al. (2010) soulignent l'intérêt qu'offre les activités en voix chantée quand les sons entendus n'existent pas dans le système phonologique de départ de la langue d'origine. La proposition de ce type d'activités peut se faire tout au début de la séance phonétique car "il s'agit de la découverte et de l'appropriation de l'outil avec lequel la langue sera ensuite observée et manipulée".

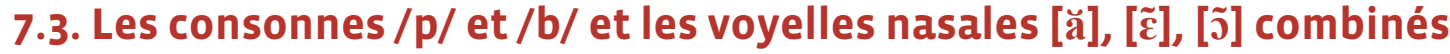

Dans notre progression pour l'acquisition de ces sons phonétiques, nous avons ensuite intégré les consonnes /p/ et /b/à la prononciation des voyelles nasales (tableau 6). Les étudiants ont dû faire attention à bien répéter l'articulation de ces deux difficultés: 


\section{TABLEAU 6}

Exercice présentant les consonnes/p/ et/b/associé aux voyelles nasales (mot monosyllabique)

\begin{tabular}{lll}
\hline$[\tilde{\varepsilon}]$ & [ä] & {$[\tilde{\mathbf{s}}]$} \\
\hline Pain & Pan & Pont \\
\hline Bain & Banc & Bon \\
\hline
\end{tabular}

Nous avons aussi entraîné les apprenants à la répétition des voyelles nasales en position inaccentuée (tableau 7).

\section{TABLEAU 7}

Exercice présentant les voyelles nasales à l'intérieur d'un mot (mot polysyllabique)

\begin{tabular}{|c|c|c|}
\hline$[\tilde{\varepsilon}]$ & [ă] & [ก] \\
\hline bien/tôt & en/tier & ton/du \\
\hline in/direct & $\tan /$ dis & con/fier \\
\hline
\end{tabular}

Puis nous avons ajouté la variation des timbres et la répétition de la voyelle nasale dans un mot (tableau 8).

\section{TABLEAU 8}

Exercice présentant les voyelles nasales d'abord en début de mot puis deux fois dans le mot

\begin{tabular}{|c|c|c|}
\hline$[\tilde{\varepsilon}]$ & [ă] & [ก] \\
\hline Informer & en partant & on s'attend \\
\hline Infini & en dînant & on s'arrange \\
\hline Inférieur & en disant & on se comprend \\
\hline Infirmier & en nageant & on se demande \\
\hline
\end{tabular}

La répétition des mots de ces trois exercices permet de s'assurer que les étudiants distinguent bien les différents phonèmes dans toutes les positions. Pour terminer, nous avons effectué des lectures de phrases regroupant cette fois-ci toutes les voyelles nasales, ce qui ajoutait une dimension prosodique:

"Attendez un instant, s'il vous plaît".

"Oh! Je vous demande pardon".

"C’est très important".

"C'est très fatigant". 
Le travail sur la prosodie et sur la respiration est crucial. La lecture d'un dialogue à haute voix permet d'entraîner les étudiants à la fluidité verbale. Afin de varier les lectures de dialogues et de textes, nous leur avons proposé de les lire seul, à deux, en groupe, chanté, murmuré, à débit lent, rapide ou hésitant, en colère, comme un robot, triste, etc., ceci dans le but d'apporter une dimension un peu théâtrale et ludique.

"Quel beau temps!"

"C'est le printemps!"

"Et c'est dimanche!"

"Un beau dimanche de printemps!"

"Quelle chance!" (Abry \& Chalaron, 2010).

De plus, un autre texte fut suggéré afin de travailler le rythme et l'intonation et les voyelles nasales. Pour cette activité, les étudiants devaient écouter le texte et l'avoir sous les yeux. Ils devaient ensuite le lire à haute voix.

Une chambre, c'est une pièce dans laquelle il y a un lit; une salle à manger, c'est une pièce dans laquelle il y a une table et des chaises, et souvent un buffet; un salon, c'est une pièce dans laquelle il y a des fauteuils et un divan; une cuisine, c'est une pièce dans laquelle il y a une cuisinière et une arrivée d'eau; une salle de bains, c'est une pièce où il y a une arrivée d'eau au-dessus d'une baignoire; quand il y a seulement une douche, on l'appelle une salle d'eau; quand il y a seulement un lavabo, on l'appelle cabinet de toilette; une entrée, c'est une pièce dont au moins une des portes conduit à l'extérieur de l'appartement; accessoirement, on peut y trouver un portemanteau; une chambre d'enfant, c'est une pièce dans laquelle on met un enfant; un placard à balais, c'est une pièce dans laquelle on met les balais et l'aspirateur; une chambre de bonne, c'est une pièce que l'on loue à un étudiant (Perec, 1974: 57-58).

Les stratégies utilisées tout au long de cette étude ont permis aux étudiants d'attirer leur at-

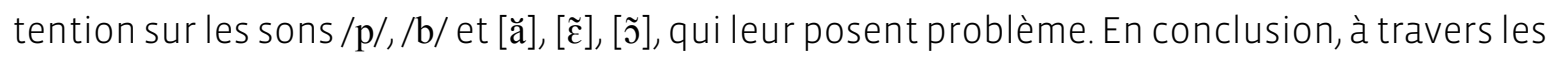
entretiens individuels effectués en fin de semestre, nous pouvons affirmer qu'une meilleure

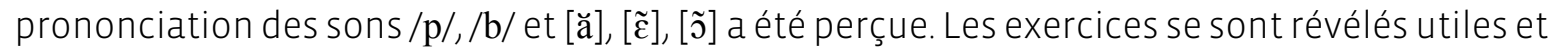
probants car nous avons observé une meilleure prononciation quand nous les avons écoutés lire ou s'exprimer en français.

Après avoir travaillé en grand groupe durant tout le semestre, il a fallu s'assurer, de manière individuelle, que le travail a porté ses fruits. Chaque élève a été enregistré et évalué par le professeur. Chaque entretien individuel a duré dix minutes et se composait de trois parties. La première partie concernait la reconnaissance et reproduction des sons phonétiques

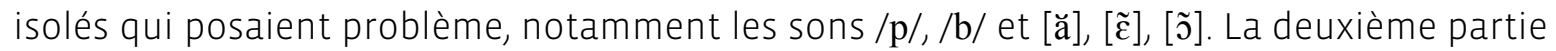
demandait aux élèves de lire des phrases écrites en alphabet phonétique. La dernière partie

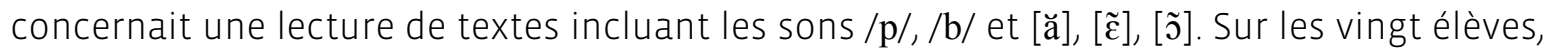
quatorze ont réalisé et reproduit ces sons de manière adéquate. 


\section{Conclusion}

Nous avons pu présenter dans les pages précédentes un ensemble d'exercices et d'activités représentant l'interaction que l'enseignant doit établir entre la connaissance des systèmes phonologiques de la langue maternelle des apprenants et de la langue étrangère apprise, la définition des difficultés des apprenants et de leur processus d'apprentissage et la connaissance des méthodes pédagogiques à sa disposition. Cette interaction permet de développer le bien-fondé de la pédagogie de la prononciation ainsi que de la correction des erreurs du français langue étrangère dans le contexte universitaire jordanien. Une formation théorique et pratique des enseignants de langue permet de dépasser les obstacles de l'enseignement de la prononciation sans la contextualiser. En effet, les manuels FLE n'offrant pas d'exercices précis destinés au public jordanien ne peuvent pas développer chez les apprenants jordaniens la prononciation de façon communicative. Nous avons présenté une méthode d'analyse des erreurs de la prononciation ainsi que certains exercices à faire. II s'agit d'un travail qui cherche à inspirer les enseignants-chercheurs jordaniens voire arabophones pour développer un manuel spécialisé en prononciation.

Pour continuer à améliorer l'enseignement de la phonétique à l'Université du Yarmouk, nous préconisons les recommandations suivantes:

Les enseignants doivent proposer des exercices et des activités variés pour motiver les apprenants. Il faut également ne pas vouloir tout corriger, mais lorsque l'enseignant s'attèle à l'étude d'un phonème, il doit veiller à corriger ce phonème en particulier pendant la séance. Les petites classes sont préférables car ayant plus de temps de parole les apprenants peuvent être davantage corrigés et plus de temps peut être consacré à chacun d'entre eux. Par la suite, comme Pagel et al. (2012) le recommandent, "[i]l s’agit en fait de donner les moyens de communiquer en situation réelle par la suite en permettant à l'apprenant de devenir de plus en plus autonome". Une formation continue des enseignants doit être régulièrement organisée pour améliorer leurs savoirs et savoir-faire en matière de l'enseignement de la phonétique et de travail sur la correction phonétique.

Bien entendu la correction phonétique ne doit pas se limiter à une classe en particulier, mais dès qu'une occasion se présente et lors des années d'apprentissage de la langue, il convient de faire de la phonétique. Enfin, nous soulignons l'intérêt de mener d'autres études sur la correction phonétique des étudiants en français en tenant compte des dialectes arabes existants dans les classes jordaniennes.

\section{Réfẻrences bibliographiques}

Abdessemed, Nadjiba, \& Samir Abdelmanmid, 2015 : "Conception d'un didacticiel pour la phonétique progressive du FLE dans l'enseignement universitaire algérien”, Synergies Algérie 22, 207-216 [disponible en file:///C:/Users/rana\%20kandeel/Desktop/modifications/abdessemed_abdelhanmid.pdf]. 
Abry, Dominique, \& Julie Veldeman Abry, 2007 : Phonétique, Paris : CLE International.

Abry, Dominique, \& Marie-Laure Chalaron, 2010 : Les 500 Exercices de Phonétique A1 A2, Paris Hachette.

ABRY, Dominique, 2008: "Phonétique française et pédagogie de la prononciation" [http://www. ac-grenoble.fr/casnav/accueil/enseigner-FLE-FLS/index.php?post/2012/07/11/Phon\%C3\%Agtique-fran\%C3\%A7aise-et-p\%C3\%Agdagogie-de-la-prononciation\#Ancreo, date de récupération : 1er février 2017].

AL-SHIHRI, Faiz, 2002: Production et perception des voyelles orales du français langue étrangère par les apprenants saoudiens, problème d'assimilation. Thèse de doctorat en Phonétique, sous la direction de Jacqueline Vaissière. Université de Paris III.

AL-TAmımı, Jalal-Eddin, 2006 : "Analyse dynamique de la réduction vocalique en contexte CV à partir des pentes formantiques en arabe dialectal et en français" in Actes des XXVIème Journées d'études sur la Parole.

Al-Tamım, Jalal-Eddin, 2007 : Indices dynamiques et perception des voyelles, étude translinguistique en arabe dialectal et en français. Thèse de doctorat, Université de Lyon 2 en France.

Al-TAmimı, Jalal-Eddin, Marion GIRARD \& Egidio Marsico, 2002 : "Variabilité inter-langue et inter-individuelle en production et en perception : étude préliminaire en arabe dialectal et en français" in Actes des XXIVème Journées d'Étude sur la Parole.

Blanchet, Philippe, 2002 : "Implications Pratiques de la Phonologie Fonctionnelle: de la Méthodologie de Recherche à l'Intervention Didactique”, La Linguistique 38 (2), 37-52.

CECRL : Consell de L'Europe, 2000 : Un Cadre Européen Commun de Référence pour les Langues: Apprendre, Enseigner, Evaluer, Strasbourg : Division des Politiques Linguistiques.

Cheddad, Bougerra, 2011: "Contribution de la phonétique à l'enseignement/apprentissage du Français dans le cycle primaire”, Synergies Algérie 14, 145-150 [disponible en https://gerflint. fr/Base/Algerie14/bouguerra.pdf].

Corder, S. Pit, 1980 : “Que signifient les erreurs des apprenants?”, Langages 57, 9-15.

Cornaz, Sandra, Nathalie Henrich \& Nathalie Vallee, 2010 : "L'apport d'exercices en voix chantée pour la correction phonétique en langue étrangère : le cas du français langue étrangère appliqué à des apprenants italiens d'âge adulte", Recherche et pratique pédagogiques en langue de spécialité (cahiers de l'APLIUT), XXIX (2) [disponible en https://apliut.revues. org/757\#tocto2n5].

Cuq, Jean-Pierre, \& Isabelle GrucA, 2005 : Cours de Didactique du Français Langue Etrangère et Seconde, Grenoble: Presses Universitaires de Grenoble. 
Galisson, Robert, \& Daniel Coste, 1976 : Dictionnaire de didactique des langues, Paris: Hachette.

MAUme, Jean-Louis, 1973: "L'apprentissage du français chez les Arabophones maghrébins (diglossie et plurilinguisme en Tunisie)", Langue Française 19, 90-107.

Pagel, Dario, Edith Madelenı \& François Wioland, 2012 : Le Rythme du Français Parlé, Paris: Hachette.

Paveau, Marie-Anne, \& Georges-Elia Sarfati, 2003 : Les grandes théories de la linguistique. De la grammaire comparée à la pragmatique, Paris : Armand Colin.

Perec, Georges, 1974 : Espèces d’Espaces, Paris : Galilée.

SAIDANe, Tahar, Mounir Zrigul \& Mohamed Ben Ahmed, 2004: "La transcription orthographique-phonique de la langue arabe", communication dans le colloque Récital.

Sung, Hee Park, 2002 : “L’oral et les Apprenants Coréens. Problèmes de Phonétique et de Culture”, Études de Linguistique Appliquée 126 (2), 201-210.

YousIF, Deya-Eldin, 1999: Réalisation des voyelles du français standard par des arabophones du Qatar: étude phonétique contrastive. Thèse de doctorat en Phonétique, sous la direction de René Gsell. Université de Paris III.

WaCHS, Sandrine, 2011: "Tendances actuelles en enseignement de la prononciation du français, langue étrangère (FLE)”, Revista de Lenguas Modernas 14, 183-196. 